\title{
Femur Fractures are a Risk Factor for Multiple Organ Failure in Critical Injured Trauma Patients
}

Justin E Richards, MD*\#; Robert V O’Toole, MD*\$

*R Adams Cowley Shock Trauma Center, \#Division of Trauma Anesthesiology, \$Department of Orthopaedics, University of Maryland School of Medicine, Baltimore, Maryland

\section{INTRODUCTION}

- Femur fractures are frequently the result of a high energy mechanism and often associated with multi-system injuries ${ }^{1}$.

- Multiple organ failure (MOF) occurs in nearly $10-15 \%$ of critically injured trauma patients and is a manifestation of the systemic immune response to severe injury 2 .

- Few studies have examined the risk of MOF in critically injured trauma patients with a femur fracture.

\section{HYPOTHESIS}

- We hypothesize that femur fractures are an independent risk factor for MOF after adjusting for concomitant organ system injuries, and depth of clinical shock.

\section{DISCLOSURES}

- The authors report no relevant financial disclosures for this study.

\section{METHODS}

- Retrospective study at a single tertiary care trauma center.

- Inclusion: all trauma patients age $\geq 18$, injury severity score (ISS) $>15$, admitted to the intensive care unit (ICU).

- Exclusion: Abbreviated Injury Scale (AIS) score head $>2$, ICU stay $<2$ days.

- Femur fracture defined as occurring between the lesser trochanter and the distal metaphyseal flare were recorded.

- MOF defined by the Denver Organ Failure score ${ }^{3}$.

- Cox proportional hazard model assessed the impact of femur fractures on MOF.

- Interaction terms tested the significance of a femur fracture and other organ system injuries (i.e. chest and abdomen).

\begin{tabular}{|c|c|c|}
\hline & HR & $95 \% \mathrm{Cl}$ \\
\hline Femur fracture & 1.88 & $1.06-3.35$ \\
\hline Age & 1.03 & $1.01-1.04$ \\
\hline Admission lactate & 1.16 & $1.08-1.23$ \\
\hline Chest_Abdominal Injury & 1.05 & $1.01-1.09$ \\
\hline
\end{tabular}

HR: hazard ratio, $\mathrm{Cl}$ : confidence interval

\section{RESULTS}

- 737 critically ill, multi-system trauma patients.

- 104/737 (14\%) sustained a femur fracture.

- MOF occurred in 70/737 (10\%) patients.

- Trauma patients with a femur fracture demonstrated an $88 \%$ increased hazard ratio (HR: 1.88, 95\% Cl 1.06-3.35) for MOF (Table 1).

- There was no significant interaction with femur fracture and chest injury or femur fracture and abdominal injury and MOF.

\section{CONCLUSION}

- Critically injured trauma patients with a femur fracture demonstrate a significantly increased risk for MOF.

- This risk is independent of concomitant organ system injuries.

REFERENCES

1. Enninghorst N, McDougall D, Evans JA, et al. Population-based epidemiology of femur shaft fractures. J Trauma Acute Care Surg. 2013;74(6):1516-1520.

2. Sauaia A, Moore EE, Johnson JL, et al. Temporal trends of postinjury multiple-organ failure: still resource intensive, morbid, and lethal. J Trauma Acute Care Surg. 2014 Mar;76(3):582-92. 3. Sauaia A, Moore EE, Johnson JL, et al. Validation of postinjury multiple organ failure scores. Shock. 2009;31(5):438-447. 